\title{
Epidemiology and the control of disease in China, with emphasis on the Chinese Biobank Study
}

\author{
L. Li ${ }^{a, b,{ }^{*}}$, Y. Guo ${ }^{b}$, Z. Chen ${ }^{c}$, J. Chen ${ }^{d}$, and R. Peto ${ }^{c}$ \\ aSchool of Public Health, Peking University, Xueyuan Road 38, Beijing, China \\ ${ }^{\mathrm{b}}$ Chinese Academy of Medical Sciences, Beijing, China \\ ${ }^{c}$ Clinical Trial Service Unit and Epidemiological Studies Unit, University of Oxford, UK \\ ${ }^{d}$ Chinese Center for Disease Control and Prevention, Beijing, China
}

\section{summary}

Similar to many other developing countries, China is facing a double burden of disease as a result of epidemiological transition. Non-communicable diseases (NCDs) represent a major challenge, having an adverse effect on the health of the Chinese population and increasing the economic burden of health care. In today's era of evidence-based medicine and decision making, China, as a developing country, has a lack of local scientific evidence which will affect the effectiveness of NCD prevention and control.

As such, and on the basis of decades of cooperation and trust with the University of Oxford, the Chinese Biobank Study [Kadoorie Study of Chronic Disease in China (KSCDC)] was commenced in 2004. KSCDC, an international prospective project, aims to establish the basis of a blood-based health database, using genetic, environmental and lifestyle aspects to investigate and understand the causes, risk factors, pathogenesis, prevalence patterns and trends of major chronic diseases in China (such as stroke, coronary heart disease, cancer, diabetes, hypertension, chronic obstructive pulmonary disease etc.). This study has a duration of 15-20 years, and will provide scientific evidence for strategic planning of NCD prevention and control, and development of new treatment and intervention approaches. In total, approximately 510,000 adults aged 30-79 years have been recruited from the general population in 10 geographically defined regions (five rural and five urban) of China, with differing disease profiles and differing risk exposures. Extensive data collection has been undertaken with questionnaires, physical measurements, and collection and storage of blood samples. KSCDC is a multi-factor, multi-disease, multi-disciplinary large-scale chronic disease epidemiological study, and is also one of the largest long-term blood-based population cohort studies ever conducted in the world. It is worth mentioning that all gene specimens are kept in China, and all associated intellectual property rights are owned by international cooperation groups; this breaks new ground for Chinese and foreign international cooperation.

\footnotetext{
(C) 2011 The Royal Society for Public Health. Published by Elsevier Ltd. All rights reserved.

${ }^{*}$ Corresponding author. School of Public Health, Peking University, Xueyuan Road 38, Beijing, China. Imlee@ pumc.edu.cn (L. Li). Competing interest

None declared.
} 
This article describes the study design, baseline description and main results to date.

\title{
Keywords \\ Epidemiology; NCD prevention and control; Chinese Biobank study; KSCDC \\ Health transitions: The need for and challenge of medical services in urban and rural areas
}

\begin{abstract}
With urbanization, industrialization and strong economic growth in China, the Chinese population is undergoing rapid and profound health transitions. The life expectancy of different population groups (e.g. Guizhou, Gansu, Beijing, Shanghai etc.) has increased with increasing gross domestic product per head. There are also disparities in health, and significant differences exist between different population groups in China. Deaths from infectious diseases and total fertility have decreased, while deaths from non-communicable diseases (NCDs) have increased. Similar to other developing countries, China is facing a double burden of disease.
\end{abstract}

\section{Disease burden: The social and economic impact of diseases}

At present, NCDs are the main health threat in China. From 1975 to 2005, mortality due to NCDs decreased from 569.0 to 523.5 per 10,000 people, while the proportion of NCDs increased from $71.1 \%$ to $80.2 \%$. In 2009 , the four leading causes of death in China were NCDs (cancer, cerebrovascular diseases, heart disease and respiratory diseases), accounting for $78.7 \%$ and $79.7 \%$ of all deaths in urban and rural areas of China, respectively. NCDs account for $68.6 \%$ of the total disease burden. ${ }^{1}$ As such, NCDs are a major public health problem and health threat for Chinese residents, accounting for over $80 \%$ of the 10.3 million deaths in China each year. ${ }^{2,3}$ Moreover, the prevalence of NCDs increased in both urban and rural areas from 1993 to 2008, and the number of people with major NCDs in China is expected to increase significantly from 2010 to $20300^{3,4}$

Compared with 1993, the economic burden of disease in China increased 6.4- fold in 2005, while GDP growth increased 4.2-fold over the same period; the cost proportion of NCDs increased from 54\% in 1993 to $65 \%$ in 2005. The total medical cost of NCDs was 1.48 trillion RMB (210 billion USD) in 2005. The proportion of the disease burden for NCDs increased from 54\% in 1993 to $63 \%$ in 2005. Approximately 3.71 million premature deaths were caused by NCDs, accounting for $75 \%$ of all premature deaths in China. ${ }^{5}$ If this trend continues, this will increase to $73 \%$ by 2020 . This invisible epidemic is an under-appreciated cause of poverty, and hinders the economic development of many countries. The global epidemic of chronic diseases must be controlled effectively.

\section{Risk factors: Focal points of disease prevention and intervention}

The choice of prevention and control strategies for NCDs in China depends on an awareness of the complicated risk factors in the aetiologic chain. NCDs have direct causes and remote causes (cause of causes). Regarding the remote causes, such as socio-economic determinants 
(social, economic, cultural, political and environmental factors), although the mechanism to causality is less clear, the available evidence is sufficient to suggest action. The three most important modifiable risk factors are unhealthy diet, physical inactivity and tobacco use. The vast majority of cases of NCDs are caused by a small number of known and preventable risk factors. As such, NCDs can be prevented by risk factor interventions.

Globally, these risk factors are increasing as people's dietary habits change to foods high in fats and sugars, and people's work and living situations are much less physically active.

Three risk factors (tobacco use, poor diet and lack of physical activity) contribute to the four major NCDs (heart disease, type 2 diabetes, lung disease and many cancers) which are responsible for more than $50 \%$ of deaths in the world. In 2005, there were economic losses totalling 300 billion RMB due to smoking [320 million active smokers, 270 million passive smokers, 67\% smoking rate in males, 30 million young smokers ( $\_0$ years)], accounting for approximately $1.5 \%$ of total GDP in 2005. According to the standards of the World Health Organization, an estimated 215 million people in China are overweight or obese. Adjusted for measures more applicable to the Asian population, that number increases to 281 million. ${ }^{6}$ The prevalence of hypertension in urban and rural areas of China increased between 1979 and 2002. ${ }^{7}$ However, the rates of awareness, treatment and control of hypertension in China are only $30.2 \%, 24.7 \%$ and $6.1 \%$, respectively. ${ }^{7}$

\section{NCD prevention and control: Practice and research}

In order to address the major challenge of NCDs, China is strengthening NCD prevention and control institutions and systems, including the Chinese Centre for Disease Control and Prevention, National Cancer Centre and National Centre for Cardiovascular Diseases, and will create a strong team for NCD control and prevention. ${ }^{6}$ The Chinese Government issued a series of policy and technical documents for NCD control and prevention, such as the National Plan for Diabetes Prevention and Control 1996-2000, the National Plan for Cardiovascular and Cerebrovascular Diseases Prevention and Control 1996-2010, the National Plan for Tumour Prevention and Control 1986-2000, the National Plan for Cancer Prevention and Control 2004-2010, the National Standard for NCD Prevention and Control, and Standards on Nutrition. The Chinese Government has also developed a series of regulations to improve unhealthy diets, physical inactivity and tobacco use in recent years, and published various professional guidelines related to obesity and overweight, hypertension, dyslipidaemia and diet, etc. For example, the Chinese Ministry of Health Bureau of Disease Control and Prevention, the National Patriotic Health Campaign Committee Office and the Chinese Centre for Disease Control and Prevention cosponsored the National Healthy Lifestyle Action.

Two research programmes on NCD prevention and control have been conducted in China, namely the Community Intervention for Health $(\mathrm{CIH})$ in Hangzhou ${ }^{8}$ and the Chinese Biobank Study. ${ }^{9}$

The CIH is a multinational programme of the Oxford Health Alliance for comprehensive community interventions, including China, India, Mexico and the UK in the pilot stage. Three main components in the design of the interventions are community coalition building, 
health communication and structural intervention. These combine to create communities in which healthy choices are easy choices. These measures will be implemented in four settings: schools, workplaces, community health centres and neighbourhoods. In addition to the $\mathrm{CIH}$ project, Hangzhou city happens to be undertaking some important changes at the same time which share the same aims as the $\mathrm{CIH}$ project to build a healthy city. One major initiative is Hangzhou public bicycle service, sponsored by the local government, which started on 1 May 2008. This complimentary bicycle rental system, as part of public transport, has been established in Hangzhou to solve the problem of the last $1 \mathrm{~km}$ from the bus stop to home or work. This programme effectively encourages active transportation, facilitates long-term behaviour changes when it comes to daily transportation, and, in turn, improve health.

The Chinese Biobank Study (Kadoorie Study of Chronic Disease in China) of 0.5 million people in China is a large-scale, long-term follow-up cohort study to enhance the quality of relevant evidence in the Chinese population. It is an open-ended study with broad research aims. A baseline survey (questionnaire, physical measurements and blood sample collection) was performed, and morbidity and mortality are being followed-up, with periodic resurveys of a sample of participants to help determine the main avoidable causes of NCDs in China. In total, approximately 510,000 adults aged 30-79 years have been recruited in 10 areas (Figs. 1 and 2). Cause-specific mortality data are obtained through death registries. Data on fatal or non-fatal events for ischaemic heart disease (IHD), stroke, diabetes and cancer are obtained through disease registries. At present, 3500 cases of IHD, 3500 cases of diabetes, 7000 strokes (75\% confirmed by computer tomography/magnetic resonance imaging) and 8000 deaths have been reported in this cohort. There will be continuous long-term follow-up for a range of fatal and non-fatal conditions. The main findings include large unexplained variations in disease rate, co-existence of poverty and affluence, relatively well-established infrastructures, and high-quality survey data, with the ability to follow-up both fatal and non-fatal events and the potential to study very different ranges of risk exposures (e.g. blood lipids, body mass index and genes). The results will be relevant to the prediction, prevention and understanding of disease aetiology in China and other countries.

In conclusion, China's population is undergoing rapid and profound health transitions, and the burden of disease is increasing significantly. The major risk factors of NCDs are out of control if measures and interventions do not conduct immediately, and the potential risks are becoming more serious. The long-term health issues of Chinese people cannot be optimistic. The concept of NCD prevention and control is gradually changing, with more attention given to remote risk factors from disease with prevention measures extended to policy, environmental and economic approaches from medical treatment. NCD prevention and control has a long way to go.

\section{Acknowledgements}

Ethical approval

The central ethics approvals were obtained from Oxford University and the China National CDC for Chinese Biobank Study. The Community Intervention for Health $(\mathrm{CIH})$ project was approved by the Institutional Review Board at Peking University Health Science Center. 
Funding

The baseline survey and first re-survey of Chinese Biobank Study were supported by a research grant from the Kadoorie Charitable Foundation in Hong Kong; follow-up of the project during 2009-14 is supported by the Wellcome Trust in the UK (grant 088158/Z/09/Z); the Clinical Trial Service Unit and Epidemiological Studies Unit (CTSU) at Oxford University also receives core funding for it from the UK Medical Research Council, the British Heart Foundation, and Cancer Research UK. Community Interventions for Health (CIH) is supported by a registered UK charity, the Oxford Health Alliance (OxHA) and facilitated by MATRIX Public Health Solutions Inc. For a full list of donors supporting OxHA please go to www.oxha.org.

\section{REFERENCES}

1. World Health Organization. Burden of disease study. 2009.

2. China Ministry of Health. Death cause surveillance from China disease surveillance points. 2005.

3. China Ministry of Health. China Nutrition and Health Survey. 2002.

4. China Ministry of Health. China national NCD risk factor surveillance. 2007.

5. Bureau of Disease Prevention and ControlChina Ministry of Health and Chinese Center for Disease Control and Prevention. Report on chronic non-communicable diseases in China. 2011.

6. Wu Y. Overweight and obesity in China. BMJ. 2006; 333:362-3. [PubMed: 16916811]

7. China National Center for Cardiovascular Diseases. Report on cardiovascular disease in China (2008-2009). Encyclopedia of China Publishing House; 2010.

8. Community intervention for health in Hangzhou. [last accessed 20.10.11] Available at: http://www. 3 four50.com/cih/china.php

9. Chen Z, Lee L, Chen J, et al. Cohort profile: the Kadoorie Study of Chronic Disease in China (KSCDC). Int J Epidemiol. 2005; 34:1243-9. [PubMed: 16131516] 


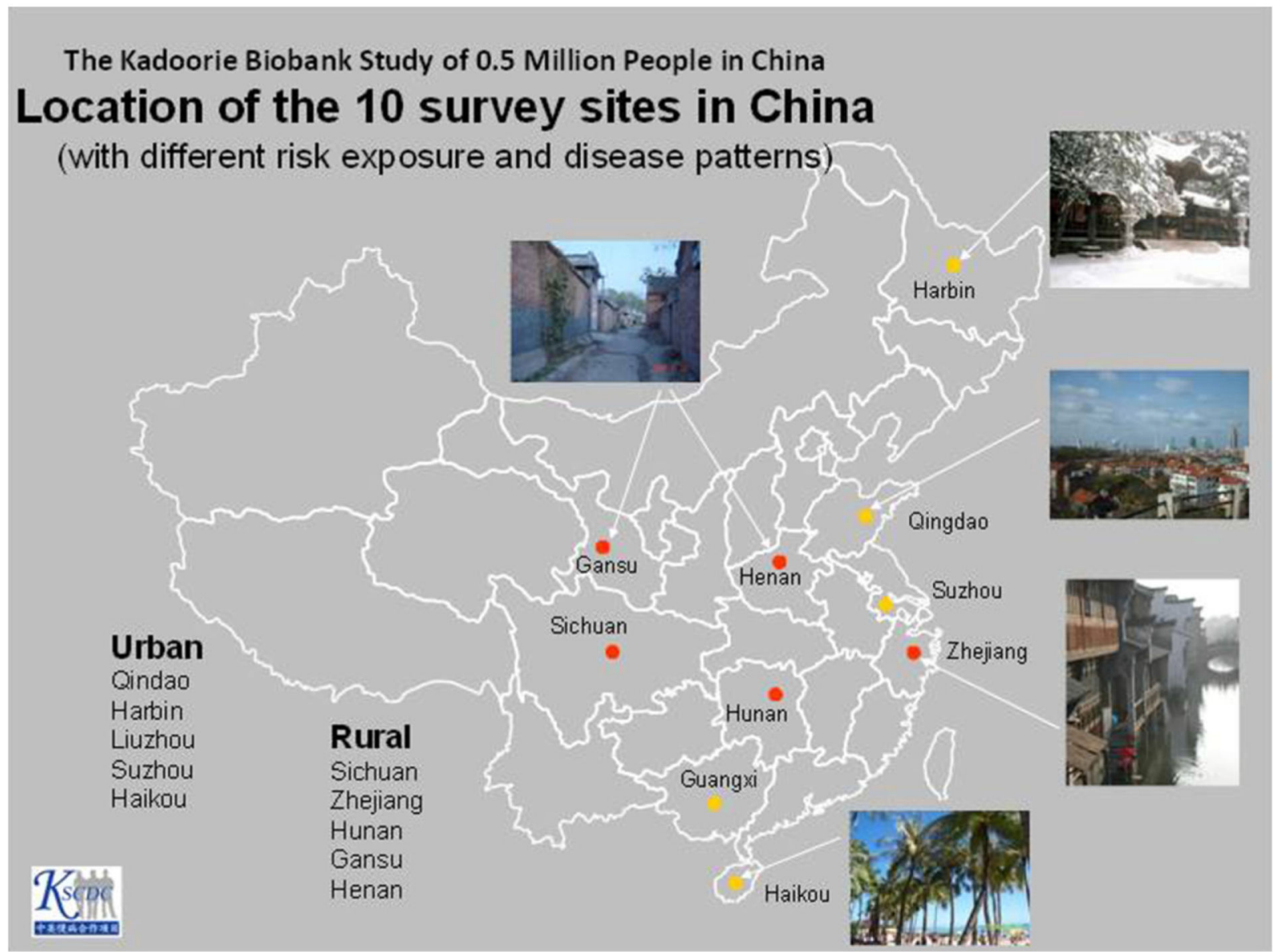

Fig. 1. The location of the $\mathbf{1 0}$ survey sites in the Chinese Biobank Study 


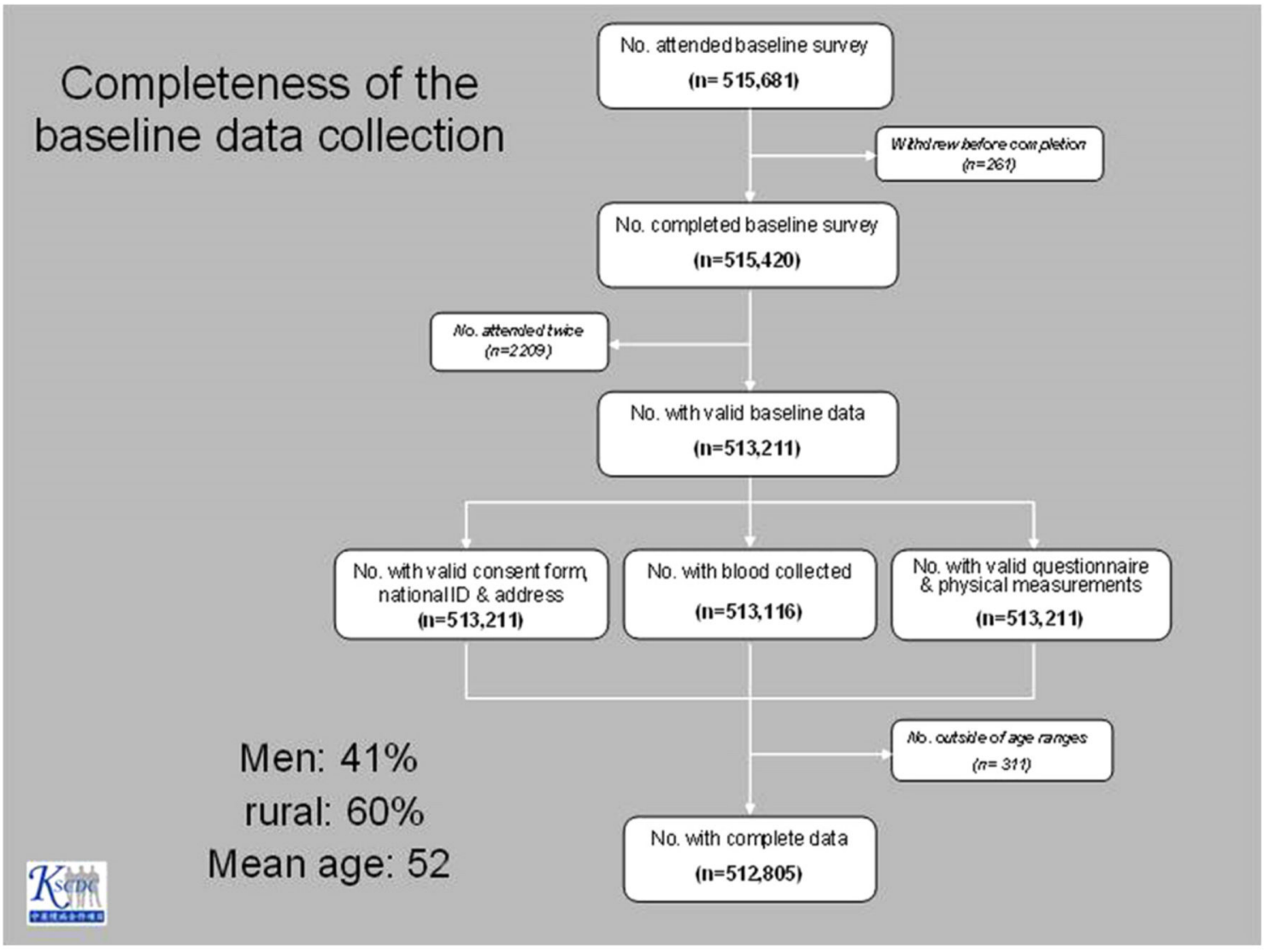

Fig. 2. The completeness of the baseline data collection in the Chinese Biobank Study. 